\title{
The rôle of control and system theory in systems biology
}

\author{
Peter Wellstead *, Eric Bullinger, Dimitrios Kalamatianos, Oliver Mason, Mark Verwoerd \\ The Hamilton Institute, National University of Ireland, Maynooth, County Kildare, Ireland \\ Received 19 November 2007; accepted 22 February 2008 \\ Available online 15 May 2008
}

\begin{abstract}
The use of new technology and mathematics to study the systems of nature is one of the most significant scientific trends of the century. Driven by the need for more precise scientific understanding, advances in automated measurement are providing rich new sources of biological and physiological data. These data provide information to create mathematical models of increasing sophistication and realism-models that can emulate biological and physiological systems with sufficient accuracy to advance our understanding of living systems and disease mechanisms.

New measurement and modelling methods set the stage for control and systems theory to play their rôle in seeking out the mechanisms and principles that regulate life. It is of inestimable importance for the future of control as a discipline that this rôle is performed in the correct manner. If we handle the area wisely then living systems will present a seemingly boundless range of important new problems-just as physical and engineering systems have done in previous centuries. But there is a crucial difficulty. Faced with a bewildering array of choices in an unfamiliar area, how does a researcher select a worthwhile and fruitful problem? This article is an attempt to help by offering a control-oriented guide to the labyrinthine world of biology/physiology and its control research opportunities.
\end{abstract}

(C) 2008 Elsevier Ltd. All rights reserved.

Keywords: Biology; Systems biology; Control systems; Systems dynamics; Estimation; System identification; Mathematical biology; Computational physiology

\section{Introduction}

\subsection{Background}

In this paper we consider the question What are the biological problems where a (control) systems approach can be useful? This is a huge question and it would be misleading to suggest that there is a definitive answer. However, a number of avenues of inquiry exist and they fall into two categories: the definite and the speculative. The definite category relates to areas in which there is already an established path for a systems approach. By contrast, the speculative category includes questions that are fundamental to understanding the mechanisms of life and where there may be an opportunity for control theory to offer useful viewpoints. This suggestion is made with caution, in the knowledge that some of the world's most

\footnotetext{
A version of this paper was originally presented at the 10th IFAC Symposium on Computer Applications in Biotechnology and the 8th IFAC Symposium on Dynamics and Control of Process Systems, Cancun, Mexico, June 2007, and uses additional material from the lectures 'Schrödinger's Legacy' and 'The Industrialisation of Biology'.

* Corresponding author.

E-mail address: peter.wellstead@nuim.ie (P. Wellstead).
}

talented life scientists have previously trod this ground. However, there are historically important examples where the physical sciences have shed new light on the mechanisms of life. And now, at the beginning of the 21 st century, there is good reason to believe that experts in the theory of dynamical systems and signals can contribute in a similar way. Indeed, later in the text we will see areas where control system analysis and theory, alongside other branches of engineering, are already providing new insights into biological phenomena.

\subsection{Commercial and scientific motivation}

At no previous peacetime period has the direction of science and technology been so strongly guided by economic forces and political agencies. Global competition between rival economies means that businesses and governments search for competitive advantages, with intellectual property derived from novel science and technology being one means of providing such advantages. This has led governments to actively manage and direct research funding in a rigorous manner. This trend, coupled with the decline of traditional technological industries, has led researchers in all areas of science to respond accordingly and, where appropriate, to seek new avenues of inquiry. 
In the case of control systems specialists, the transfer of their skills from physical systems to living systems is an obvious idea. The problem is that the transfer is not straightforward. Biology is not just another application area of standard control theory - the language, culture and (most of all) the nature of the systems is very different. Taken together, these issues present a significant intellectual challenge to control systems researchers trained in technological ways. It is nevertheless a challenge that the control community has accepted as it attempts to make the transition from existing to new sources of important and useful research tasks. An aim of this article is to help in the transition by indicating the differences between how biologists consider problems and how they sub-divide the topics. This is then followed by an outline of the aspects of systems biology as seen from the life science perspective and the areas where opportunities exist for the control researcher to gain access to interesting and relevant life science problems.

\subsection{Systems and life science: technical differences}

The life sciences cover an enormous range of systems in scale (temporal and spatial), function and type. The scope of behaviour starts with the minute workings of molecular machinery and culminates in the behaviour of the coordinated system of organs and tissue that forms an organism. In between are cellular systems in which signals are processed, and the hierarchies of organised assemblies of cells which form tissue types and organs. Beyond the single organism is the study of populations of organisms and their joint behaviour.

Engineers are by training accustomed to systems with a hierarchy of size and temporal scale. In biology however the scale and range of the hierarchy is staggering. For example, human physiological function emerges from the orchestrated behaviour of around 30,000 genes, more than 100,000 protein and over 200 cell types. Moreover, these elements function at timescales ranging from microseconds at the molecular level, to years in the life span of an organism.

In the engineering world, the purpose of system design is to coordinate the operation of components so that they contribute in a harmonious way to the overall performance of a system. The same harmonious coordination seems to exist in living systems, but with a crucial difference-living organisms are not of our construction and we can only theorise on how they came to be as they are. With no design blueprint, we lack an objective frame of reference against which to assess their performance-a performance which is in any event highly complex and requires a level of analysis that needs to be more critically attuned than in physical systems. For example, living systems can be both robust to internal variations in some conditions and yet sensitive to small changes at other times. This resilience/sensitivity to changes has interesting parallels in feedback control where robustness and fragility are opposite faces of the same coin (Dorato, 1998; Keel \& Bhattacharyya, 1997). But the complexity and strong nonlinearity of living systems render parallels with technology potentially misleading; such parallels often being no more than the human habit of rationalising the unknown in terms of past certainties. More will be said of this later in Section 6 .

\subsection{Systems and life science: cultural differences}

The apparently unfathomable complexity of living systems caused the biological sciences to adopt a 'reductionist' approach. That is to say the biological quest for the understanding of an organism begins by examining the rôle and function of the smallest indivisible component. This information is then used in a 'bottom-up' attempt to infer the properties of the organism as a whole. Reductionism has served us well in the past, but the times are changing and the use of 'bottom-up' biology as a means of understanding overall function is under fire. For example, it is the essence of the Lazebnik's (2002) humorous criticism in Can a Biologist Fix a Radio?, while the same point is made more seriously in Strange (2005), and with passion in Noble (2006).

The value of reductionism is now openly questioned. But there is an equally questionable cultural aspect to life science research that is rarely challenged. This relates to the view of biology as a 'pure' discovery-based science in which systems method imported from applied sciences can play only a subservient rôle. The tension that this creates between pure science and the systems approach has been an interesting, and sometimes disturbing, ${ }^{1}$ sociological side-show to the growth of systems biology, and is an indicator of the cultural gulf that still separates traditional biology from a system-driven approach. Paradoxically, it is also this tension that gives systems biology its potential power, since by the analysis of systems as a connected set of components - operating at different levels - a new dimension is added to life science. In this spirit, the multilevel systems approach to analysis is a particularly powerful complement to traditional life science research methodologies and the reductionist tradition.

\subsection{General literature and references}

A distinctive form for a systems approach to biology is developing through the ideas of dynamical systems methods, multi-level analysis and the study of complexity. The content of this article is intended to add some specific features to this emerging form as it regards control systems studies. Before proceeding however, it is good to note that the idea of a systems approach is gaining credence in biology. At the moment this is mainly through the benefits of mathematical modelling as a way of capturing biological knowledge in a quantitative and objective form (Di Ventura, Lemerle, Michalodimitrakis, \& Serrano, 2006; Mogliner, Wollman, \& Marshall, 2006). There are also books (Alon, 2006; Palsson, 2006) that cover the growing area of systems biology in its various complexions. New books emerge regularly, with the book by Klipp, Herwig, Kowald, Wierling, and Lehrach (2005) being a particularly good and comprehensive introduction. In the engineering control journals the review paper by Sontag (2005) is an excellent guide for control theorists interested in cellular

\footnotetext{
${ }^{1}$ In words that may return to haunt him, Plasterk (2005) has described systems biology as a form of 'scientific pornography'.
} 
signalling. Another source worth mentioning is the upcoming joint special issue (Vidyasagar, Khammash, \& Tomlin, 2008). The journal IET Systems Biology (2006) is an important outlet for publications in this area, and there are a number of edited volumes available which illustrate the range of views and approaches that exist (Alberghina \& Westerhoff, 2005; Bringman, Butcher, Parry, \& Weiss, 2007; Kitano, 2001).

As background, it is also important to note the long history of mathematical biology (Murray, 1989; Rubinow, 1975) and mathematical physiology (Keener \& Sneyd, 1998). These topics predate the current growth of interest in a systems approach, and are important sources of detail modelling of many fundamental mechanisms. In this spirit, it is important that we acknowledge the debt that is owed to the pioneers of biochemistry, mathematical biology and physiology research. Their work underpins current systems research in biology and physiology.

\subsection{Organisation of the paper}

As indicated in Fig. 1, the article has two parts: Part I discusses the various areas that, taken together, make up systems biology as a subject. Part II then considers the control systems opportunities that arise from these areas. Part I is laid out as a biologist, physiologist or medical practitioner would see things. As a result, the separation between topics may appear at first sight to be arbitrary. For example, metabolism and cell signalling are mutually overlapping processes-both are concerned with coupled sets of chemical reactions. Nonetheless, they are considered separately, since this is how they are usually treated. Likewise, the idea of homeostasis, which is intimately linked to control of the metabolism, is presented in the context of its medical origins.

These inconsistencies apart, some effort has been made to arrange items in Part I from a control perspective. Specifically in
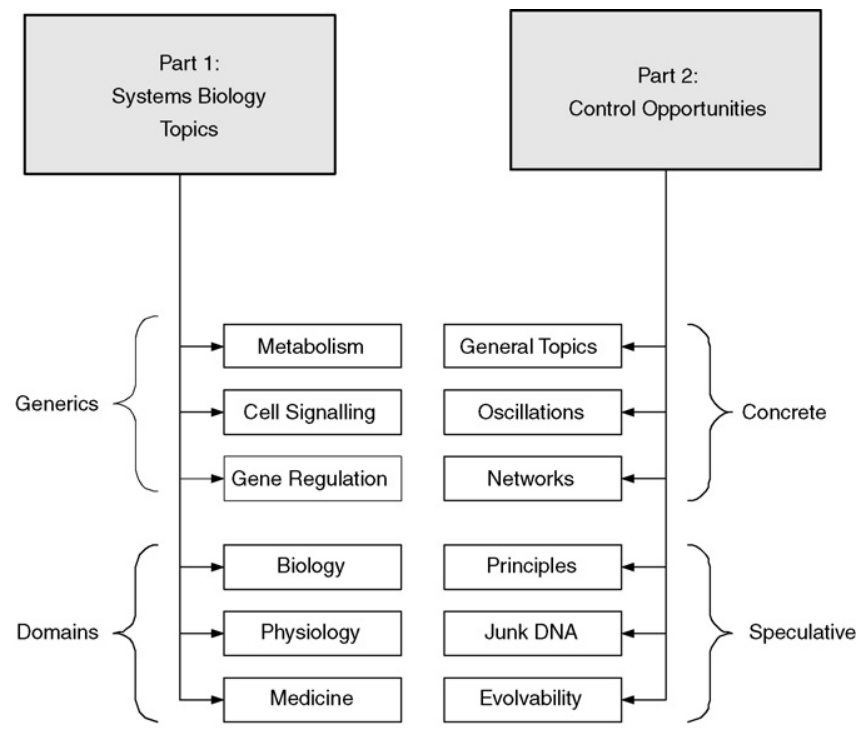

Fig. 1. Layout of the paper, showing the subdivision of areas from the viewpoint of the life scientist (left hand side) and the control systems scientists (right hand side). control systems analysis it is usual to distinguish between the flow of material and energy through a system and the information processing components that regulate the system. There is a loose parallel in living systems, where the flux of chemicals and energy that sustains life in an organism as a whole is studied as metabolism and the processing of information within a cell as cell signalling. ${ }^{2}$ In this spirit, metabolism (Section 2) and the transmission of information within the cell (Section 3) are used to open Part I. This material then provides the background to their use in systems approaches to physiology (Section 4), and medicine (Section 5).

Part II is straightforwardly arranged in two sections: Section 6 covers some of the specific control opportunities as they relate to the material in Part I. Section 7 describes the more speculative areas of life science where a control theoretic approach may help illuminate areas that are as yet largely unknown. The article closes with some reflections on the rôle of mathematical modelling and renewal in science.

\section{Part I: topics in systems biology}

Here we are concerned with research topics as they are perceived and classified in the systems biology community. As indicated previously, the sub-divisions that have emerged are not systematically arranged. Nevertheless for the purposes of this article, and its cross-referencing to the systems biology literature, the accepted classifications are retained.

\section{Metabolism}

Metabolism: the totality of all chemical transformations carried out by an organism.

Thus is metabolism described by Harold (2001) in his layperson's guide to cell biology. In this article however we distinguish between the transformations that are primarily about material flux and energy, and those that concern the transmission of information. Thus to Harold's definition, we add the codicil: where the primary purpose is the transport, transformation and use of bio-molecular material to sustain life.

Each metabolic process consists of a large number of interconnected sets of enzyme-catalysed biochemical reactions. The set of reactions form a network of biochemical pathways which work in concert to achieve the metabolic purpose. The dynamic behaviour of a metabolic network evolves as a function of the enzyme kinetics associated with each reaction and their interconnection through the network. At a qualitative level, metabolism is well understood by life scientists. However, the quantitative reasons for networks taking particular forms, and the corresponding metabolic dynamics, are not well known. Thus although every college text on physiology and medicine deals with metabolism in detail

\footnotetext{
${ }^{2}$ Note: The equally important area of gene regulation is, for reasons of space, omitted completely from this review.
} 
(Guyton \& Hall, 1996), many challenges remain before we can claim a quantitative understanding of metabolic dynamics and structure. From this viewpoint, the area of metabolism is a good entry point for control systems analysts, particularly control engineers with a chemical engineering background who will already be familiar with the biochemical processes involved. In the following sections this familiarity is assumed, and only the key points are reviewed.

\subsection{Reaction kinetics}

The mathematical modelling of metabolism uses the law of mass action applied to the metabolic reaction sequence diagram and combined with the corresponding relations for individual reactions. This statement contains the two key aspects of metabolism-(a) the modelling of the individual reactions and (b) the analysis of the networks formed by coupled reactions to achieve the metabolic purpose. Consider first the reactions: enzymatic reactions (Cornish-Bowden, 2004) are complex chemical processes leading to nonlinear state space models. Various simplifying assumptions can be made with regard to the enzyme kinetics (Vera, Balsa-Canto, Wellstead, Banga, \& Wolkenhauer, in press), with the Michealis-Menten (Michealis \& Menten, 1913) quasi-steady state approximation being the most frequently used. Even after such approximations, the model of the metabolic process remains nonlinear and relies upon either linearising assumptions for their solution, or simulation for visualisation (Klipp et al., 2005).

\subsection{Metabolic networks analysis and control}

The networks of coupled reactions formed by a metabolic process are often large and complex. However a great deal can be learnt by considering the equilibrium conditions of the reactions. These correspond to a system of linear equations with coefficients given by the stoichiometry of the various reactants. The solutions to the network equations provide information on steady state fluxes, and by adding chemical/thermodynamic constraints, a great deal of information can be obtained about the operation of the metabolic process (Varma \& Palsson, 1994).

Yet more steady state information is obtained using metabolic control analysis (MCA) (Fell, 1997; Heinrich \& Rappaport, 1974). This much studied area is not about control in the usual sense, but the analysis of how fluctuations in one part of a network can influence other parts. Specifically, it relates to the sensitivity analysis of concentrations of molecules and reaction rates in a network to small parameter changes, i.e. changes of enzyme concentrations. This is extremely useful for processes in homeostatic equilibrium (see Section 5.1), since it provides insights into the local sensitivity of a metabolism in normal steady state operation. This said, the idea of a set of steady state levels being the norm in metabolism is not generally true, and dynamical studies are of increasing importance (Ingalls \& Sauro, 2003). Of particular relevance is the multi-scale nature of metabolism-multi-scale dynamics is a common feature in biological processes and more will be said of it later.

\section{Cell signalling}

An attractive and well-established area of systems biology for control researchers is the modelling and analysis of the sequences of chemical reactions which cause information to be transmitted within a cell. It is easy to see why the cell is a basic functional element of all organisms and its operational mechanisms are fundamental to all aspects of biology. From a systems viewpoint, the cell is interesting because it is a closed environment, with distinct input ports and response mechanisms. The input ports are the receptor sites in the cell membrane where signalling molecules may attach and initiate coupled sets of chemical reactions (signalling pathways) within the cellular space (Fall, Marland, Wagner, \& Tyson, 2002).

Signalling action through the pathways result in a cellular response which can take a number of forms (Downward, 2001) including proliferation, cell death and cell differentiation. The modelling of these pathways is discussed in Section 3.1. The dynamical models of signalling pathways are nonlinear and the analysis of their behaviour is challenging and problematic, this is discussed in Section 3.2. A number of chemical reaction sequences are found repeatedly in biology and there is a considerable effort to characterise them as distinct modules, this characterisation is considered in Section 3.3.

\subsection{Cell signalling pathway models}

Intracellular signalling paths are less well known than extracellular metabolic paths, and so typically, 'what-if' simulations are used to probe the rôle of various components of a signalling pathway. This in itself is a big step forward for biologists for whom intracellular experiments are time-consuming, with results that are frequently unreliable and unrepeatable. The possibility of rapid, cheap and exactly repeatable in silico simulation of cellular signalling mechanisms represents a breakthrough in the study of cell biology (for example see Heinrich, Neel, \& Rappaport, 2002; Neves \& Iyengar, 2002).

Despite rapid advances, the area is, for various reasons, still in its infancy. For example, the biological 'picture-models' of cell signalling pathways are often wrong or incomplete. Also, there are complexities in the representation of signal pathways caused by 'cross-talk' between different pathways and the multiplicity of interacting signalling mechanisms. An indication of the scale of complexity can be gained by glancing at the current 'best' picture of the toll-like receptor signalling network (Oda \& Kitano, 2006)(this network is vital to the immune system's response). Despite the complexity of this model, and the great care associated with its assembly, the representation is still incomplete, possibly incorrect in parts and gives only a topological picture of the receptor signalling network.

\subsection{Analysis of cell signalling pathway models}

As noted in the preceding sections, the use of mathematical models in biology involves many approximations-some are known and others unknown. Even if the current generation of mathematical models were to be structurally correct, there are 
issues of model accuracy, calibration and validation. For example, the reaction coefficients are generally unknown and must be either estimated, guessed, or taken from the literature. In the same spirit, the structure of a mathematical model is dependent upon the biologist's current beliefs of what constitutes the correct signalling pathway-these are often incomplete or incorrect. Indeed, one reason for mathematical modelling is to test proposed structures, and some of the most useful contributions by systems biologists have been to suggest new signalling pathway structures which were subsequently verified by actual experimentation. The uncertainty in parameters and structure means that there is a need for parameter estimation and structural determination methods to be developed for the forms of model found in cell signalling. As a result system identification for signalling pathway models is an area where needs exist. These are discussed later in Section 6.1.

Intracellular measurements are generally not possible, and those that are possible are difficult and error prone. As a result, in many cases it is extremely difficult to estimate the coefficients of a signalling pathway model. In this situation the special structure of the system dynamics might be used to infer properties, as in for example positive/non-negative matrices and monotone systems (Angeli \& Sontag, 2003)(see Section 6.1). In practice however, it has been the use of computer simulations of dynamical models using parameters from the literature that has shown immediate practical benefit. For examples of such investigative modelling, see the work on the MAP kinase pathway (Asthagiri \& Lauffenburger, 2001; Kolch, 2000).

\subsection{Functional modules in signalling pathways}

Just as in metabolic flux pathways, the structural analysis of signalling pathways offers many opportunities for creative connections with what we know from technological control theory. A particular issue is understanding the biological function of various parts of a pathway. Typically, and allegedly because of the exploratory nature of evolution, the functions of different parts of a signalling pathway are not obvious. Indeed some parts may have no apparent rôle in the cellular function under investigation. A further aspect of this is the multifunctional nature of biological processes whereby a part of a pathway may only come into play under certain special circumstances. One extremely worthwhile objective for control systems analysis is to determine the functions performed by different parts of a system and when they perform them (Schmidt \& Jacobsen, 2004). A number of researchers believe that the key to such functional assignment is the encapsulation of frequently recurring patterns of bio-molecular reactions into motifs that serve specific functions (Alon, 2006; Tyson, Chen, \& Novak, 2003). As an example, a widely occurring structure is the protein phosphorylation cycle (Kholodenko, 2006).

\section{Physiology}

The previous section covered the systems associated with intracellular signalling and metabolic flux pathways. Physiology is concerned with the way biological structures are actuated and animated by these pathways. Specifically, we consider the systems approach to the cellular assemblies, tissues and organs that work together to form entire organisms. Taking the meaning of 'system' in its broadest sense, physiology is by definition a systems approach to how organisms function. The addition of mathematical modelling and other methods from the engineering sciences has taken physiology from focusing on a general description of function to a level that involves great mathematical and computational sophistication. The dynamical and control systems problems found at this level are qualitatively different from metabolic flux pathways and intracellular signalling, since in addition to bio-chemical dynamics, they also explicitly include the large-scale features of an organism, such as electrical, mechanical and/or hydraulic effects. This area should be of interest to members of the control systems community who specialise in the integration of sub-system mathematical models into models of entire systems. A particular challenge here is that the mathematical modelling crosses all disciplines, and the integration of model components spans the complete range of temporal and spatial scales.

\subsection{Modelling of organ function}

Initially, mathematical physiology (Keener \& Sneyd, 1998) was concerned with developing mathematical models of specific physiological phenomena. Over time this has moved toward integrating models of individual physiological elements into computer-based models of organ functions, graduating over the years into mathematical models of entire organs. The virtual heart project (Noble, 2002) was one of the earliest among such projects and is the most advanced. Starting with a mathematical model of cellular electrical potentials (Noble, 1962), the virtual heart project has progressed into a large multi-partner international collaboration. A number of other virtual organ projects have started in recent years with aims ranging from visualisation for education, training and surgical rehearsal to fundamental investigation of disease and organ malfunction. Most of these projects operate under the umbrella of an ambitious physiological modelling project-The Physiome Project.

\subsection{The Physiome Project}

The aim of the Physiome Project is to provide a coherent framework for the mathematical modelling and analysis of human physiology. In particular, through the various projects that compose the Physiome Project (Hunter \& Borg, 2003) international teams are developing systematic ways in which to apply a systems engineering approach to physiological processes. This area is a rich one for further development as its benefits become clear and it attracts the attention of commerce and clinicians (Friedland, 2004). The complexity of the Physiome Project means that the mathematical modelling and analysis of physiological processes associated with it are progressing as part of an international effort using common repositories of knowledge and integrated programmes (Hunter, 2005). 


\subsection{Computational tools for systems biology}

There is a wide range of computational tools for systems biology, and (Alves, Antunes, \& Salvador, 2006) is a recent review. However, for metabolic and cell signalling dynamics, research focused upon specific pathways and their dynamical and structural properties, then the standard scientific computing tools of MATLAB, Octave or Scilab are appropriate. In particular, while the systems of equations involved in signalling and flux pathway models are strongly nonlinear and often of high dimension, they are usually within the capability of standard scientific simulation methods. This approach is further supported by systems biology 'toolboxes' specifically designed for widely accepted scientific computing environments, such as MATLAB (Mathworks, in press; Schmidt, 2006; Schmidt \& Jirstrand, 2006). Thus, when relatively small groups of computer literate researchers are involved, and the models are of reasonable complexity, then exchanging and understanding models is not a problem. The difficulties lie in analysing the performance of the model and making biological sense of the performance.

As mathematical models become accepted in the life science community, then methods are required with which to transmit the model to different formats for scientific languages and computational tools. With this in mind, the Systems Biology Markup Language (SBML) (Hucka et al., 2003; SBML, 2006) was developed as a method of exchanging quantitative biological information. It does this by providing a means of capturing research results and modelling developments in a format usable by a large number and variety of systems biology application programmes. In the words of the SBML website:

"The systems biology community needs information standards if models are to be shared, evaluated and developed cooperatively. SBMLs widespread adoption offers many benefits, including: (1) enabling the use of multiple tools without rewriting models for each tool, (2) enabling models to be shared and published in a form other researchers can use even in a different software environment, and (3) ensuring the survival of models (and the intellectual effort put into them) beyond the lifetime of the software used to create them."

SBML is targeted specifically at systems of ODEs and nonlinear algebraic equations in biochemical networks. The alternative language cellML does the same, but is intended to be more general in that it encodes any system of ODEs/algebraic equations in MathML and then gives the model components biological meaning through the metadata (Hunter, Li, Mc Culloch, \& Noble, 2006).

As a final point on computational tools, we note that the time and effort needed to assemble mathematical models, and determine the numerical values of their coefficients, has led to the sharing of models. As a result, mathematical models can increasingly be found online. An overview of model databases can be found on Institute of Systems Biology (2006), with particular examples: BioModels Database (2006), JWS Online
(2006), the Physiome Project (2006) and the cellML model repository cellML Model Repository (2006).

\section{Systems medicine}

Medical practice is necessarily cautious and conservative in nature. Since antiquity, the field has evolved gradually through a combination of observational methods and diagnosis based upon the particular experience and training of individuals. Unfortunately, doctors generally have only a basic knowledge of mathematics. As a result, the quantitative methods that are available for medical decision-making are too often ignored or misunderstood (Sutherland, 1992). This has meant that the gradual move to more systematic and mathematically based techniques in biology and physiology are only slowly touching medicine. But touching it they are, and this section discusses the ways in which this is happening.

\subsection{Homeostasis}

The biological and physiological perspectives of systems biology are highly important. There is however a complementary viewpoint based on the part performed by feedback control in living systems as it is perceived in medicine. It relates directly to metabolic control (Section 2) and has a bearing on the potential practical uses of systems biology in physiology, medicine and pharmacology.

Building on ideas that date back to the Greek philosophers, the distinguished French physiologist Claude Bernard (18131878) argued that compensatory internal physiological processes exist within an organism that work to balance externally imposed disturbances. Bernard's practical observations spoke clearly of feedback regulation and disturbance rejection mechanisms within living organisms (Bayliss, 1966). These ideas were further codified in the 1950s by Cannon (1932) in his book The Wisdom of the Body. With extensive historical references (to Hippocrates and Pflüger amongst others) and based on extensive research, Cannon was able to give a range of practical examples of feedback regulation in physiology. More importantly, he gave it a name-homeostasis. Since Cannon's time other researchers (Sterling, 2004) have expanded upon the homeostatic principle of internal physiological control in important ways and homeostasis has become a fundamental principle in medicine and physiology (see for example Tortura \& Grabowski, 2003).

\subsection{Personalised and predictive medicine}

The systems approach to metabolism, cell signalling, physiology, and medicine are different routes that converge on a common aim-an improved understanding of the mechanisms of life and disease. The four have a specific meeting point in the area of personalised medicine (Weston \& Hood, 2004). The personalised medicine proposition is that mathematical modelling and a systems approach, combined with high-throughput biological measurement technology, can supply the analytical tools and patient bio-marker data for an 
individual approach to patient care. Within this framework, genetic information and proteomic time histories will allow treatments to be individually tailored and systematically administered. Likewise, a time history of an individual patient's bio-markers will allow predictive statements to be made with regard to future health states, and enable corresponding preventative actions to be taken. This aspect of systems medicine has been variously referred to as personalised (The Royal Society, 2005), predictive and/or preventative (Hood, Heath, Phelps, \& Lin, 2004) medicine. The pharmaceutical companies have yet another interpretation, in which drug treatments would be 'personalised' for specific subgroups of the population.

Personalised medicine implies a significant shift from what might be termed population-based medical care, where the emphasis is on average/typical responses to disease. In personalised medicine, data from population responses will form a backdrop against which to assess information on how a specific individual behaves in a diseased state, and subsequently responds to treatment. For this to be viable, several ingredients may be needed such as:

- More regular monitoring, including self-monitoring, of important diagnostic indicators.

- Enhanced tools for learning appropriate individual information from time trends of individual diagnostic indicators.

Like personalised medicine, predictive medicine represents a marked change from current practice. Specifically, it is a move from reactive medical care to one in which an individual's susceptibility is predictively treated. In particular, the use of statistical inference and statistical prediction from bio-marker records would allow the forecasting of the onset of a condition, and the use of preventative measures.

\section{Part II: opportunities for control}

This part is divided into two sections. The first (Section 6) outlines areas in which control systems analysis can contribute in a clear and well-defined manner. The second (Section 7.1), describes more speculative topics where dynamical systems theory may play a part.

\section{Control systems analysis in biology}

Control studies in biology have existed for many years, with a convenient time-stamp being the book (Wiener, 1948). There was enormous respect for Wiener and this created an enthusiastic following for his ideas of using communications and control systems to describe biological phenomena. Like Cannon, Wiener also invented a name that captured the imagination-cybernetics. The name cybernetics was at first a blessing, it gave identity to the transfer of control and communication ideas from technology to biology. Unfortunately, a well defined scientific agenda failed to emerge for cybernetics and it was not until later that the relevance of control in a systems approach to biology began to take shape.
This occurred when ideas of dynamics and control were combined with well-founded mathematical models of biological function. This took initial forms in cell signalling pathway analysis (see Section 3.2), in quantitative understanding of metabolism (see Section 2) and related topics described in Part I. Subsequently, a wide range of opportunities have emerged for the transfer of control theoretic principles to the systems that make up living organisms. It is the aim of this section to summarise these, with Section 6.1 covering the areas already mentioned and Sections 6.2 and 6.3 describing important areas not directly covered thus far.

\subsection{Review of control topics in systems biology}

In this section we collect the aspects of the systems biology topics covered in Part I and outline the associated control systems opportunities.

\subsubsection{Metabolic control analysis}

The study of the metabolism is well established and is thus a good entry point for the control analyst. Likewise, the mathematical machinery for MCA (Reder, 1988) is framed in a similar way to control system sensitivity analysis and thus presents familiar territory. Also, as remarked in Section 2, steady state analysis is insufficient for a full understanding of metabolic processes. In this vein, MCA has recently been explicitly expressed in a control systems format and extended to the dynamical case (Ingalls, 2004) in a way which opens other control theoretic opportunities. This is an important step since, as is emphasised elsewhere in this article, it is the dynamical analysis of complexity in biological systems that will allow control systems analysts to add constructively to biology.

\subsubsection{Modelling of signalling pathways}

From a pragmatic viewpoint, mathematical modelling and computer simulation are the most clearly identifiable successes of systems biology. The current modelling procedures described in Section 3.1 are first generation methods that have shown the potential of mathematical modelling and 'what-if' computer simulation. However, as the need to account for different chemical reaction conditions grows, so too will the need for new modelling approaches. For example, stochastic models are required where there are few molecules involved (Turner, Schnell, \& Burrage, 2004), while power-law models are suitable when there is molecular crowding (Vera et al., in press). Likewise, almost all current signalling modelling neglects the spatial and molecular transport aspects of the signalling sequence. This simplification has been acceptable in these early days of modelling of cell signalling when even models containing gross approximations are proving informative, but it cannot endure. There are thus very significant opportunities for mathematical modellers with a control systems and dynamics background to contribute. For an authoritative guide to the state of the art and future possibilities see Kholodenko (2006). 


\subsubsection{Motifs and functional modules}

Related to the issues of mathematical modelling is the area of functional blocks in biological processes. The idea of motifs and functional modules (as described in Section 3.3) is very attractive from a control systems viewpoint (Hartwell, Hopfield, Leibler, \& Murray, 1999; Tyson et al., 2003; Zhang et al., 2005) as it enables functional blocks to be grouped together and the signalling process made more comprehensible. The way in which control systems analysts can contribute is by determining the complete dynamical performance of these motifs. The idea of assigning specific function to assemblies of biochemical reactions in this way is seductive. It relates to research into the function of biomolecular objects (e.g. genes, proteins, etc.) in biological networks (see Section 6.3), and at a more abstract level to speculation concerning general control and organising principles in biology (see Section 7.1). From a practical viewpoint, a considerable amount of work has been done on identifying functional modules. Nonetheless, there is more to be added, particularly from the viewpoint of metabolic and signalling networks and their linkage to multi-level/multiscale control systems.

\subsubsection{Feedback systems in biology}

As hinted earlier in this article, nature apparently uses negative feedback so that a robustness-sensitivity compromise exists. Control specialists have analysed examples of feedback control and written extensively on biological robustness and sensitivity in living organisms (e.g. Csete \& Doyle, 2006; Ingalls, 2004; Kitano, 2004). Kitano (an influential figure in systems biology) interprets robustness more broadly than the control theoretic meaning. Other authors draw directly upon results in classical control (Bode, 1945; Nyquist, 1932). In particular, Stelling, Sauer, Doyle, and Doyle (2004) and Yi, Huang, Simon, and Doyle (2000) have been prominent in developing the biological perspectives of the control concepts of robustness and fragility (Dorato, 1998). These discussions of biological robustness are scientifically important for a number of reasons. Not least because of their strategic value for the future of control engineering ideas in the life sciences. Specifically, they have raised the profile of control theory by showing life scientists how a control systems analysis can provide a theoretical underpinning to experimental observations (Bullinger, Findeisen, Kalamatianos, \& Wellstead, 2007).

Within the cell, feedback (positive and negative) is an important structural feature of signalling networks. Regulatory mechanisms of negative feedback have already been mentioned, however positive feedback loops also occur in biological circuits in a way that have oscillator, bi-stable and gating analogues in electric circuits (Millman \& Taub, 1956). While such basic ideas and many examples of biological feedback have been developed, this area remains a rich one, both for control theoretic work and for particular biological examples. In particular, and despite the excellent work already referred to, the concept of robustness is still not sufficiently well formulated in a biological context.

\subsubsection{Transient performance and nonlinearity}

This area is in essence related to the previous item, but is treated separately because of the biological significance of stability and transient performance. The issue of stability (particularly instability induced by positive feedback) was also mentioned in the previous item and is raised again in Section 6.2. The discussion here however is different, since it relates to the nonlinearities and multiple timescales which occur in biological system dynamics, and the implications that these features have for transient performance. For example, in connection with nonlinear response (Wolkenhauer, Mesarović, Sreenath, Wellstead, \& Rolfs, 2006) demonstrates how changing initial conditions and the pattern of external excitation will dramatically change the observed performance of a nonlinear biological system. As the cited paper remarks:

"It would take an experimental biologist many timeconsuming and expensive experiments to obtain comparable results under each operating condition. And then there would be no way of determining that all the results were generated by the same biological mechanism."

The clarification of nonlinear dynamics in this and related ways can be of enormous benefit in unifying apparently unrelated experimental observations and thereby generalising experimental results. Here mathematical control theory can play a powerful part in elucidating fundamentals (see for example the work of Angeli and Sontag, 2003)

As mentioned earlier (Section 4), the wide range of timescales in biological responses means that the analysis of transient response must have a multiple timescale aspect. In analytical terms this means that there may be no steady state operating point in the classical sense. As a result there is scope for control systems experts to fruitfully work on new stability measures for multi-scale systems and descriptions of the temporal behaviour of specific nonlinear biological phenomena.

\subsubsection{Special system structures}

In intracellular systems biology, the continuous time dynamics of signalling pathways are often combined with the essentially logical machinery of gene expression. Together with transport delays in protein synthesis, this may lead to hybrid systems with time delay properties that are exceedingly difficult to analyse (Henzinger \& Sastry, 1998). It has proven possible to graphically analyse some specific low dimensional hybrid systems (see for example Pettit \& Wellstead, 1997). In general, however, the area is difficult and the level of analysis is either very abstract, or very specific to the case in hand. In an effort to make the abstract analysis less general, there is a systems analysis opportunity to consider the specific hybrid forms that occur in signalling pathway dynamics (Downward, 2001).

Dynamical descriptions of metabolic flux and cell signalling lead to positive systems. This can potentially be used to advantage since positivity gives special properties to temporal behaviour, and then enables the analyst to make useful 
performance statements with limited knowledge of parameter values. This is useful because of the difficulty in estimating numerical values for reaction kinetics. As noted previously, monotone systems theory for systems biology has been pioneered by Sontag (2005) and Angeli and Sontag (2003).

\subsubsection{System identification and estimation}

Thus far, much of system identification work in this area has involved applied mathematicians, see for example the review (Crampin, Schell, \& McSharry, 2004). However, because of their prior experience with parameter and structure estimation for complex dynamical systems, system identification researchers from the control systems community can add a further dimension to the area. The starting point for this should be a recognition that biology is not merely an application for standard identification methods. Genuinely new approaches are required that recognise (i) the specific types of nonlinear, timevarying processes found in living organisms (Bullinger, Findeisen, \& Streif, 2006), (ii) the particular form of measurements that are available (Klipp et al., 2005) and (iii) the numerous disturbances and measurement errors that are specific to biological processes and sensing.

With these caveats, there is great scope for system identification experts to develop signal processing and identification methods that are specific to the biological application. There are many challenges, including methods to

1. Extract meaningful information from biological/medical sensing modalities.

2. Deal with unusual measurement errors and disturbances.

3. Handle unconventional data forms and data collection protocols.

4. Incorporate unusual forms of prior information and constraints.

\subsubsection{Getting quantitative information from measurements}

The previous paragraph mentioned that the measurement methods available in biology are quite specific. This bears expanding upon because, compared to the measurement and sensing of physical systems, the measurement of variables in living systems is either difficult, crude or not possible. In addition, biology has historically focused primarily on gathering qualitative data and has only recently begun to report quantitative results. The difficulty in biological measurements means that there are significant opportunities for new sensor data analysis procedures and sensor data fusion methods to be developed. Also, new technical advances from engineering fields could allow for the generation of more quantitative biological data and feature extraction tools. This is an area where instrument engineers and computer scientist are very active, but the scope is vast and the opportunities correspondingly large.

\subsubsection{Modelling and computational tools}

As described in Section 4.3, the Systems Biology Markup Language is widely used as a format for representing models in a machine transferable way. There are many software tools and modelling packages that support SBML_over 100 at the last count. For the control systems specialist the most attractive of these tools are the Systems Biology Toolbox (Schmidt \& Jirstrand, 2006) and the commercially available SimBiology Toolbox (The Mathworks, in press), both for use with MATLAB. These toolboxes are a good starting point in the modelling of biological systems, and have the usual scope for extension.

Despite a wealth of tools and packages, as shown in Alves et al. (2006), the area of computational tools for biology is one of growth. The underlying modelling principles on the other hand are less well studied. In this spirit, one potential area for research is the development of common modelling and model analysis tools which import the expertise from dynamical systems modelling. For example, systems biology is an area where systematic methods for model development and analysis, such as bond graphs, could make useful new contributions as they have done in the physical world (Wellstead, 1979).

Modelling tools for large-scale biological or physiological systems might also benefit from software-oriented control experts in hierarchical techniques developed for total plant management and enterprise control. This may even inform the multi-level/multi-scale systems biology modelling described in Hunter et al. (2006). In this vein, any control systems researcher with a strong interest in computational aspects of systems biology modelling is strongly recommended to refer to the detailed Physiome Project Roadmap (Hunter, 2005). Hunter's comprehensive and informative description of the Physiome Project makes the roadmap an essential working reference.

\subsubsection{Control and predictive medicine}

Thus far the personalised/predictive medicine described in Section 5.2 has focused on the static case where high throughput measurements are used with regular sampling of personal bio-markers to design therapy and predict outcomes using regular feedback from the bio-markers. However, a moment's thought is sufficient to see that such an approach must take into account the dynamics of internal homeostatic feedback loops. Without a proper understanding of the physiological and biological dynamics associated with a patient with a specific condition, then personalised/predictive medicine may be self-defeating (Middleton \& Wellstead, 2006).

More generally, the use of a quantitative and more technologically based approach to medical investigation and practice has attracted a great deal of interest in medical circles (for example Ahn, Tewari, Poon, \& Phillips, 2006a, 2006b). Nonetheless progress will be slow and with many organisational issues to address. The difficulty of obtaining and sharing patient records, for example, means that this area will need a structured ethical code for data handling. The primary ways in which control systems specialists can assist here are in the areas of mathematical signal processing and data handling. For example, the design of predictive methods for disease trends from the high throughput data and bio-marker sources is a clear area where random signal processing can be of use. However the data sources are distributed, inconsistent and unreliable. 
Thus the methods of data fusion and validation (used in sensor data integration) and secure control networks (from military and enterprise control and management) may be a starting point for the control community.

\subsection{Oscillations in living systems}

The harmonic analysis of signals is basic to the way in which we analyse physical systems and design technological systems. We use frequency domain methods to analyse and explain the physical world, and the frequency domain is an essential tool in communications and control systems technology. Given the universal nature of harmonic behaviour in physical systems, it is therefore natural that we ask whether living systems employ frequency modulation for communication and control. The answer is an unequivocal 'yes', and so we devote an entire subsection to parallels between frequency domain properties of technological systems and our understanding of biological signalling and function.

For example, oscillatory behaviour is believed to be important for communication in neurological systems, (see Schitztler \& Gross, 2005, and the references therein, and Varela, Lachaux, Rodriguez, \& Matinerie, 2001). In particular, the frequency selectivity represented by the distinct $\alpha$, $\beta, \delta$ and $\gamma$-bands is apparently used to achieve distinct signalling and communications objectives. This suggests that information is passed at several frequencies as a method of differentiating between different brain signalling functions. Additionally, because neural connections are formed by dense groups of connections, the issue of synchronisation (or the lack of it) within a group is important (Ioannides, Poghosyan, Dammers, \& Streit, 2004; Spencer et al., 2003). Thus frequency and phase of oscillations appear to be used to encode neural information, while the absolute amplitude is apparently less important.

The brain is a well-known example of a biological subsystem in which it is believed that frequency sensitivity is used in intercellular communication. Actually, oscillatory behaviour is everywhere in living systems (Glass, 2001). Moreover it has a determining rôle in many biological outcomes from the circadian rhythm, to the control of heart beat and beyond to the internal functioning of a cell (Nelson et al., 2004). For example, in Dolmetsch, Xu, and Lewis (1998) and Li, Llopis, Whitney, Zlokarnik, and Tsien (1998), frequency sensitivity is shown at the intracellular level. By externally modulating the frequency of oscillations in intracellular calcium it is shown that the level of gene expression is a function of frequency. This raises an interesting parallel with technological systems in which frequency modulation is routinely used to encode information. The pursuit of this idea from a systems viewpoint has radical implications for how we probe the biological function of proteins and genes. Specifically, analysis of biological function is currently performed on averaged 'steady state' data. If gene expression is sensitive to oscillations in the cytoplasm, then genetics is currently only looking at the origin on the frequency spectrum of protein/gene function. This has implications for the network ideas discussed in Section 6.3.
Let us take this point further. A fundamental feature of physical systems and their components is that they exhibit a response/output that varies depending upon the frequency of the stimulus. Frequency dependence is an expression of the dynamical nature of physical systems, thus the observation that responses of biological systems may also be frequencydependent suggests a potentially useful analogue between technical and biological components. In this context, it is interesting to recall that classical biology and physiology are concerned with the steady state behaviour of organisms. For engineers versed in systems dynamics, there is the exciting possibility of using frequency domain ideas in biology. The analysis of oscillatory processes in living organisms is led by the works of Winfree (1980) and Goldbeter (1996). Thus, there is already a rich literature on oscillations in biology as seen from a mathematical (Goldbeter, Gonze, Houart, Lepour, \& Halloy, 2001) and control systems theory perspective (Stan \& Sepulchre, 2007). There remains however many stimulating opportunities for the harmonic analysis of biological phenomena.

\subsection{Network methods: assigning function via structure}

The Human Genome Project marked a turning point in the evolution of systems biology. Specifically, the use of technology and automation to increase the rate and reliability of biological data capture and analysis was crucial to performing the sequencing in a reasonable time. The speed and repeatability of automated high-throughput analyses of biological samples was fundamental to the project's success. It gave the means to rapidly and efficiently perform the many experiments required in the Human Genome Project, thereby supplanting traditional manual laboratory methods with systematic automation. To this day for many biologists, the automation of biological measurement is systems biology.

The technological lessons learnt from the Human Genome Project were not wasted upon the scientists involved. It was a natural extension to use high-throughput (e.g. automated) methods to search for, and associate biological function with, individual genes and proteins. This is important to biologists since the Human Genome Project showed remarkable similarities between the human genome and that of other species. Thus finding special complexities in the relationships between genes (and the proteins that they coded for) in generating biological function was seen a potential way of distinguishing us as a species. An issue here is that the genome only represents a small fraction of the total DNA, and thus a valid question is 'What function does the remaining DNA have?' This is pursued later in Section 7, so for the moment we stay with the fundamental dogma that genes code for proteins and proteins are the basic elements of biological function.

Graph theory has emerged as an important tool in the search for biological function, with new approaches (Barabasi \& Oltvai, 2004; Estrada, 2006; Mangan \& Alon, 2003) being used to explain features of gene/protein interaction networks. In particular, graph theoretical techniques have been used to address several key problems in the post-genomic era including: the annotation of proteins and genes of unknown 
function (Sharan, Ulitsky, \& Shamir, 2007); linking the importance of a gene or protein to its position within the interaction network of an organism (Jeong, Mason, Barabasi, \& Oltvai, 2001; Wuchty \& Stadler, 2003); and the prediction of protein-protein interactions and experiment design (Lappe \& Holm, 2004; Valencia \& Pazos, 2002). A major issue with the use of these techniques, and what makes this area interesting for the control systems analyst, is that functional genomics and proteomics is done with only partial data (e.g. samples from the whole interaction network) and noisy information. The graphs which show interconnections between objects contain only a sub-set of the possible interconnections, and measurement difficulties mean that even those interconnections may be false due either to systematic or random measurement errors. There is a clear need for a thorough understanding of the impact of these issues on the techniques described here if they are to be reliably applied to real data (Borgatti, Carley, \& Krackhardt, 2006; Stumpf, Wiuf, \& May, 2005). In addition, and vitally important for the control systems analyst, the dynamic and contextual properties of gene and protein interaction are not accounted for.

There is an interesting comparison here with the mid-20th century developments of network theory for complex dynamical electrical networks (Balabanian \& Bickart, 1969). Although biological networks are far more complex, there is good reason to believe that traditional network dynamics will offer insights additional to the static network view. Thus with most of what is published being rapidly superseded by new experimental observations or theories, this whole area is ripe for theoretical analysis from a network dynamics viewpoint. For a current summary of this area see the survey paper (Mason \& Verwoerd, 2007).

\section{Mechanisms of life}

In this section, we consider some interesting fundamental issues in biology where a control systems viewpoint may offer a different and helpful perspective. These are problems which have big prizes for the right answers, and there is reason to believe that a view from outside the confines of biology may be needed. After all, and as noted previously, many of the most significant breakthroughs in biology have been made by outsiders. For example, Mendel studied physics at the University of Vienna, and Delbrück, Schrödinger, Crick, and Wilkins all had physics backgrounds. More generally, there is ample historical evidence that a view from outside a subject's conventions leads to new results (Christensen, 1997). With this in mind, we indicate some possible topics for study by control specialists.

\subsection{General principles in living systems}

Our quantitative understanding of the physical world is based upon a family of general principles and laws that underlie the behaviour of physical systems and processesNewton's Laws, Relativity Theory and so on. Not surprisingly a strong motivation for theorists is to find correspond- ing general principles that will explain living organisms (Casti, 2004). Within control systems theory, for example some general principles from feedback control have been applied to successfully explain a number of observed properties of organisms. (An example, is the analysis of the movement of organisms in response to chemical gradients, Bray, 2002). There is a strong consensus however that a straightforward mapping of current control theories on to biological problems is not enough, and researchers are proposing a range of alternatives with the following as examples:

- Organising principles. Mesarović and Takahara (1972) have been prominent in developing a theory of multi-level systems in order to determine general organising principles for biology. The power of the multi-level concept is that it accounts for much of what is missed in other functional theories for biology. For example, in proteomics the straightforward association of protein groups with particular functions is flawed since it does not account for the context (or level) in which the proteins are operating.

- Modelling frameworks. The multi-level systems theory of Mesarović provides one possible way of developing organising principles in living organisms. This paradigm is appealing since it recalls the form of descriptions used in physical systems, and as such it appeals to the unity of science. Similar remarks can be made of modelling frameworks that describe biological principles. As an example, consider Wolkenhauer and Hofmeyr's (in press) model of self-organisation in cellular life. This approach brings together the issues of adaptation and development of functional blocks in biology (Section 3.3).

- Language and mathematical formalism. The previous two items represent attempts to build a theory for living systems using language that would be familiar to a mathematical physicist or engineer. However, others argue that we need to re-formulate the theoretical study of living systems by changing the language of discourse. In practical terms this means alternative mathematical formalisms that can encompass the complexity, diversity and contextual adaptation that, as we have seen, are the essence of living systems. For example, MacFarlane (2006) has suggested that ideas of agency might be used, and explores the theme in a wide ranging paper (MacFarlane, 2003). In a related context, we note that computer scientists are also addressing this area using formal logic (Mardare \& Priami, 2005). It seems likely that the world of control systems dynamics and computer science will converge in this area.

\subsection{Beyond the human genome}

The scientific world was surprised when the human genome was found to contain so few components and that a large number of these components are shared with other species. Thus the human genome is in itself insufficient to explain the complexity and variety of human life, and the subsequent research thrust in genomics has been an attempt to explain this puzzle. There are however a number of possibilities (Noble, 
2006) associated with information that lies beyond the genome ${ }^{3}$ but one has a special resonance for engineers. It goes as follows: the genome is accounted for by a small percentage of the total DNA sequence. The remaining noncoding DNA is generally considered to be 'junk' left over from many generations of random evolutionary steps.

To an engineer the more plausible theory is that the noncoding DNA is associated with the operation and regulation of gene expression. In this theory the genome is the 'set of parts' for a system, and the non-coding DNA provides the assembly and operating instructions for those parts. This analogy gains credence when one compares the manner of operation of coding DNA sequences with the action of formal computer code (Amos, 2006). A prominent proponent of this viewpoint is Mattick (Gibbs, 2003) whose explanation of non-coding DNA resembles the language of digital computer control (Mattick \& Makunin, 2006). This and other features of non-coding DNA are susceptible to a systems interpretation that offer exciting research opportunities for the control theorist.

\subsection{Evolvability of living systems}

This is an area that holds many opportunities for dynamical systems theory (Nowak, 2006) for the following reasons. The basic tenet of the Theory of Evolution (Darwin, 1998) is that evolution occurs through a series of random mutations. However, the practical evidence of evolution is that once useful features of an organism have arisen, they are preserved in subsequent evolutionary stages. The eukaryotic cell and distinct organs are examples of useful evolutionary developments that are preserved features. The implication is that evolution proceeds not by a series of truly random mutations, but is constrained in a way whereby components are not subject to change in future evolutionary steps if they are beneficial to the organism.

The idea of preserved features is taken to the cellular level in Kirschner and Gerhart (1998), from a molecular and genetic viewpoint, and elsewhere from a systems viewpoint (Freeman, Kozma, \& Werbos, 2001). The idea of constraint within a process of sequential improvement immediately causes a control theorist to think of constrained stochastic optimisation techniques. Thus, detailed biological issues apart, it should be possible to study constrained evolutional systems from the control theoretic viewpoint of constrained optimal control. In this framework stochastic populations of objects change in response to changes in environment and within constraints prescribed by the principle of retained function. This approach would offer a theory that included retention of evolved features, and could potentially offer insights into the underlying organising principles of Section 7.1.

As a codicil to this subsection, the idea of there being general organising principles underlying the mechanisms of life occurs in all areas of biological systems research. Concepts of evolvability, emergent behaviour, context-dependent adapta-

\footnotetext{
${ }^{3}$ Beyond the DNA code itself there are yet more possibilities in the area known as epigenomics (Beck \& Olek, 2003).
}

tion and robustness occur in different interpretations. These range from the view in Kitano (2004) of robustness (Section 6.1 ) as an organising principle rather than a property endowed by structure, to the biological reading of adaptation in Kirschner and Gerhart (1998) and evolvability of motifs mentioned in Section 3.3(Voigt et al., 2006). These ideas overlap in ways described in, for example, Wagner (2005). Any control theorist venturing into this area will need to read widely and deeply. The intellectual challenges are enormous, but the reward could be important to our understanding of life.

\section{Conclusion}

\subsection{On models}

The systems biology approach to living organisms can be considered as part of the ongoing process of unification within science in which mathematical models have played a central rôle (Gribbin, 2002). Newtonian physics presented mathematical rules which, when combined with the ideas of many other great scientists, gave the means to build deterministic mathematical models of the physical world-models that were later enriched by Relativity Theory. Quantum physics then gave a model of atomic structure which laid down the physical basis for chemistry. In a similar vein, astrophysics presents models of the structure and composition of the universe and how elements are formed, and our understanding of carbon-based molecules provides models of how life could arise.

Considered from this viewpoint, the systems approach to biology, with its current focus on the development of mathematical models and their analysis, is a logical sequel to the sequence of research that established the mathematical foundations for modern physics and chemistry. In the context of biology, the wonderful thing about a mathematical model is that it gives an objective quantitative format within which to embed and collate experimental observations, and then test the validity of biological hypotheses. By harnessing the power of modern scientific computation, such models provide a means for analysis and the organisation of information across a range of different and complex topics. In this spirit, a mathematical model can become the repository of shared knowledge and a focus for the interdisciplinary team work that systems biology requires.

\subsection{On change}

As mentioned in Section 1, the complexity of life means that applying a systems approach to biological processes is vastly more difficult than any problem that the physical and technological worlds have yet presented. Thus, progress in systems biology will be slow and by steady increments. The complexity of life will require us to check many directions and establish activities in many research areas. The aim of this article has been to show in general where research is already going, and in particular to indicate some research areas suitable for those control systems expert who elect to change to systems biology. 
Changing one's area of intellectual pursuit is hard in the modern research environment where constant productivity is demanded, but in the case of control systems research it is timely. The fact is that control as a research discipline has long reached maturity. The excitement of the early days has faded and there is a need for renewal-a situation that makes Goethe's maxim (Seehafer, 1999) appropriate:

Becoming older means entering on fresh business. All circumstances change and one must completely stop acting or take a new rôle with deliberation and awareness.

\section{Acknowledgements}

The preparation of this article was supported by Science Foundation Ireland under Research Professor Award number 03/RP1/I382.

The text has benefited from helpful comments and corrections from Peter Hunter, Sir Alistair MacFarlane CBE, Mike Mesarović and Jan Van Schuppen.

\section{References}

Ahn, A. C., Tewari, M., Poon, C.-S., \& Phillips, R. S. (2006a). The limits to reductionism in medicine. PLOS Medicine, 3, 709-713.

Ahn, A. C., Tewari, M., Poon, C.-S., \& Phillips, R. S. (2006b). The clinical applications of a systems approach. PLOS Medicine, 3, 956-960.

Alberghina, L., \& Westerhoff, H. V. (Eds.). (2005). Systems biology: Definitions and perspectives. Berlin: Springer.

Alon, U. (2006). An introduction to systems biology: Design principles of biological circuits. Chapman and Hall.

Alves, R., Antunes, F., \& Salvador, A. (2006). Tools for kinetic modeling of biochemical networks. Nature Biotechnology, 24, 667-672.

Amos, M. (2006). Genesis machines: The new science of biocomputing. Atlantic.

Angeli, D., \& Sontag, E. D. (2003). Monotone control systems. IEEE Transactions on Automatic Control, 48, 1684-1698.

Asthagiri, A. R., \& Lauffenburger, D. A. (2001). A computational study of feedback effects on signal dynamics in a mitogen-activated protein kinase (MAPK) pathway model. Biotechnology Progress, 17, 239-277.

Balabanian, N., \& Bickart, T. (1969). Electrical network theory. John Wiley and Sons.

Barabasi, A. L., \& Oltvai, Z. N. (2004). Network biology: Understanding the cell's functional organisation. Nature Reviews. Genetics, 5, 101-113.

Bayliss, L. E. (1966). Living control systems. English University Press.

Beck, S., \& Olek, A. (Eds.). (2003). The epigenome: Molecular hide and seek. Weinheim: Wiley-VCH.

BioModels Database. (2006). www.biomodels.net.

Bode, H. W. (1945). Network analysis and feedback amplifier design. Van Nostrand Company.

Borgatti, S., Carley, K., \& Krackhardt, D. (2006). On the robustness of centrality measures under conditions of imperfect data. Social Networks, 28, 124-136.

Bray, D. (2002). Bacterial chemotaxis and the question of gain. Proceedings of the National Academy of Sciences, 99, 7-9.

Bringman, P., Butcher, E. C., Parry, G., \& Weiss, B. (Eds.). (2007). Systems biology: Applications and perspectives. Springer.

Bullinger, E., Findeisen, R., Kalamatianos, D., \& Wellstead, P. (2007). System and control theory furthers the understanding of biological signal transduction. In I. Queinnec, S. Tarbouriech, G. Garcia, S.-I. Niculescu (Eds.), Biology and control theory: Current challenges, Vol. 357 of lecture notes in control and information sciences (LNCIS) (pp. 123-135). Berlin, Germany: Springer-Verlag.
Bullinger, E., Findeisen, S., \& Streif, R. (2006). Relating cross gramians and sensitivity analysis in systems biology. In Proceedings of the international symposium on mathematical theory of networks and systems (pp. 437-442).

Cannon, W. B. (1932). The wisdom of the body. WW Norton.

Casti, J. L. (2004). The theory of metabolism-repair systems. Applied Mathematics and Computation, 28, 113-154.

cellML Model Repository. (2006). http://www.cellml.org/models.

Christensen, C. M. (1997). The innovator's dilemma. Harvard Business School Press.

Cornish-Bowden, A. (2004). Fundamentals of enzyme kinetics. Portland Press.

Crampin, E. J., Schell, S., \& McSharry, P. E. (2004). Mathematical and computational techniques to deduce complex biochemical reaction mechanisms. Progress in Biophysics and Molecular Biology, 86, 77-112.

Csete, C., \& Doyle, J. (2006). Reverse engineering of biological complexity. Science, 295, 1664-1669.

Darwin, C. (1998). Origin of the species. Wordsworth Editions.

Di Ventura, B., Lemerle, C., Michalodimitrakis, K., \& Serrano, L. (2006). From in-vivo to in-silico biology and back. Nature, 443, 527-533.

Dolmetsch, R. E., Xu, K., \& Lewis, R. S. (1998). Calcium oscillations increase the efficiency and specificity of gene expression. Nature, 392, 933-936.

Dorato, P. (1998). Non-fragile controller design: An overview. In Proceedings of the American control conference (pp. 2829-2831).

Downward, J. (2001). The ins and outs of signalling. Nature, 411, 759-762.

Estrada, E. (2006). Virtual identification of essential proteins within the protein interaction network of yeast. Proteomics, 6, 35-40.

Fall, C. P., Marland, E. S., Wagner, J. M., \& Tyson, J. J. (Eds.). (2002). Computational cell biology. New York: Springer.

Fell, D. A. (1997). Understanding the control of metabolism. Portland Press.

Freeman, W. J., Kozma, R., \& Werbos, P. J. (2001). Biocomplexity: Adaptive behavior in complex stochastic dynamical systems. Biosystems, 59, 109-123.

Friedland, D. H. (March 2004). The virtual heart. pp. 62-68. Technology review.

Gibbs, W. W. (2003). The unseen genome: Jems among the junk. Scientific American, 289, 46-53.

Glass, L. (2001). Synchronization and rhythmic processes in physiology. Nature, 410, 277-284.

Goldbeter, A. (1996). Biochemical oscillations and cellular rhythms: The molecular bases of periodic and chaotic behaviour. Cambridge University Press.

Goldbeter, A., Gonze, D., Houart, G., Lepour, J.-C., \& Halloy, J. (2001). From simple to complex oscillatory behaviour in metabolic and genetic control networks. Chaos, 11, 247-260.

Gribbin, J. (2002). A history of science. Penguin Books.

Guyton, A. C., \& Hall, J. E. (1996). Textbook of medical physiology (Ninth ed.). WB Sanders Company.

Harold, F. M. (2001). The way of the cell. Oxford University Press.

Hartwell, L. H., Hopfield, J. J., Leibler, S., \& Murray, A. W. (1999). From molecular to modular cell biology. Nature, 402, 47-52.

Heinrich, R., \& Rappaport, T. A. (1974). A linear steady state treatment of enzymatic chains. European Journal of Biochemistry, 42, 89-95.

Heinrich, R., Neel, B. G., \& Rappaport, T. A. (2002). Mathematical models of protein kinase signal transduction. Molecular Cell, 9, 957-970.

Henzinger, T. A., \& Sastry, S. (Eds.). (1998). Hybrid systems: Computation and control. Berlin: Springer-Verlag.

Hood, L., Heath, J. R., Phelps, M. E., \& Lin, B. (2004). Systems biology and new technologies enable predictive and preventative medicine. Science, 306, 640-643.

Hunter, P. (March 2005). IUPS Physiome project roadmap. Technical report, University of Auckland. www.physiome.org.nz/roadmap/roadmap-mar05.

Hunter, P. J., \& Borg, T. K. (2003). Integration from proteins to organs: The physiome project. Nature Reviews. Molecular Cell Biology, 4, 237-243.

Hunter, P. J., Li, W. W., Mc Culloch, A. D., \& Noble, D. (2006). Multiscale modelling: Physiome project standards, tools and databases. Computer, 39, 48-54.

Hucka, M., Finney, A., Sauro, H. M., Bolouri, H., Doyle, J., Kitano, H., et al. (2003). The systems biology markup language (SMBL): A medium for representation and exchange of biochemical networks. Bioinformatics, 419, $523-531$. 
IET Systems Biology. (2006). www.ietdl.org/IP-SYB/.

Ingalls, B. P. (2004). A frequency domain approach to sensitivity analysis of biochemical systems. Journal of Physical Chemistry B, 108, $1143-1152$.

Ingalls, B. P., \& Sauro, H. M. (2003). Sensitivity analysis of stoichiometric networks: An extension of metabolic control analysis to non-steady state trajectories. Journal of Theoretical Biology, 222, 23-36.

Institute of Systems Biology. (2006). www.systems-biology.org/001/.

Ioannides, A. A., Poghosyan, V., Dammers, J., \& Streit, M. (2004). Real-time neural activity and connectivity in healthy individuals and schizophrenia patients. NeuroImage, 23, 473-482.

Jeong, H., Mason, S., Barabasi, A., \& Oltvai, Z. (2001). Lethality and centrality in protein networks. Nature, 411, 41-42.

JWS Online. (2006). jjj.biochem.sun.ac.za.

Keel, L., \& Bhattacharyya, S. (1997). Robust, fragile or optimal. IEEE Transactions on Automatic Control, 42, 1098-1105.

Keener, J., \& Sneyd, J. (1998). Mathematical physiology. Springer.

Kholodenko, B. N. (2006). Cell-signalling dynamics in time and space. Nature Reviews. Molecular Cell Biology, 7, 165-176.

Kirschner, M., \& Gerhart, J. (1998). Evolvability. Proceedings of the National Academy of Sciences, 95, 8420-8427.

Kitano, H. (Ed.). (2001). Foundations of systems biology. Boston: MIT Press.

Kitano, H. (2004). Biological robustness. Nature Reviews. Genetics, 5, 826837.

Klipp, E., Herwig, R., Kowald, A., Wierling, C., \& Lehrach, H. (2005). Systems biology in practice. Wiley-VCH.

Kolch, W. (2000). Meaningful relationships: The regulation of the RAS/RAF/ MEK/ERK pathway by protein interactions. The Biochemical Journal, 351, 289-305.

Lappe, M., \& Holm, L. (2004). Unraveling protein interaction networks with near-optimal efficiency. Nature Biotechnology, 22(1), 98-103.

Lazebnik, Y. (2002). Can a biologist fix a radio? Cancer Cell, 2, 179-182.

Li, W., Llopis, J., Whitney, M., Zlokarnik, G., \& Tsien, R. Y. (1998). Cellpermeant cased insp $_{3}$ ester shows that $\mathrm{Ca}^{2+}$ spike frequency can optimise gene expression. Nature, 392, 936-941.

MacFarlane, A. G. J. (2003). Information, knowledge and the future of machines. Philosophical Transactions of the Royal Society A, 361, 1616-1681.

MacFarlane, A. G. J. (2006). Private communication.

Mangan, S., \& Alon, U. (2003). Structure and function of the feed-forward loop network motif. Proceedings of the National Academy of Sciences, 100(21), 11980-11985.

Mardare, R., \& Priami, C. (2005). Logical analysis of biological systems. Fundamenta Informaticae, 64, 271-285.

Mason, O., \& Verwoerd, M. (2007). Graph theory and networks in biology. IET Systems Biology, 1, 89-119.

Mattick, J. S., \& Makunin, I. V. (2006). Non-coding RNA. Human Molecular Genetics, 15, R17-R29.

Mesarović, M. D., \& Takahara, Y. (1972). Multilevel, hierarchical, systems theory. Academic Press.

Michealis, L., \& Menten, M. L. (1913). Kinetik der Invertinwirkung. Biochemische Zeitschrift, 49, 333-369.

Middleton, R., \& Wellstead, P. E. (February 2006). On intervention in feedback loops with integral control: Implications for feedback medicine. Technical report, Hamilton Institute.

Millman, J., \& Taub, H. (1956). Pulse and digital circuits. McGraw Hill.

Mogliner, A., Wollman, R., \& Marshall, W. F. (2006). Quantitative modelling in cell biology: What is it good for? Developmental Cell, 11, 279-287.

Murray, J. D. (1989). Mathematical biology. Springer-Verlag.

Neves, S. R., \& Iyengar, R. (2002). Modelling of signalling networks. BioEssays, $24,1110-1117$.

Nelson, D. E., Ihekwaba, A. E. C., Elliott, M., Johnson, J. R., Gibney, C. A., Foreman B. E., et al. (2004). Oscillations in NF- $\kappa$ B signalling control the dynamics of gene expression. Science, 306, 704-708.

Noble, D. (1962). A modification of the Hodgkin-Huxley equations applicable to Purkinje fibre action and pacemaker potentials. Journal of Physiology, $160,317-352$.
Noble, D. (2002). Modelling the heart: From genes to cells to the whole organ. Science, 295, 1678-1682.

Noble, D. (2006). The music of life. Oxford University Press.

Nowak, M. (2006). Evolutionary dynamics. Harvard University Press.

Nyquist, H. (1932). Regeneration theory. Bell System Technical Journal, 11, 126-147.

Oda, K., \& Kitano, H. (2006). A comprehensive map of the toll-like receptor signalling network. Molecular Systems Biology, 2.

Palsson, B. O. (2006). Systems biology: Properties of reconstructed networks. Cambridge University Press.

Pettit, N. J., \& Wellstead, P. E. (1997). Analysis and redesign of an antilock brake system controller. IEE Proceedings of the Control Theory and Applications, 144, 413-426.

Physiome Project. (2006). www.physiomeproject.org.

Plasterk, R. (2005). Q and A. Current Biology, 15, R861-R862.

Reder, C. (1988). Metabolic control analysis: A structural approach. Journal of Theoretical Biology, 135, 175-201.

Rubinow, S. I. (1975). Introduction to mathematical biology. John Wiley and Sons.

Systems Biology Markup Language (SBML). (2006). www.sbml.org.

Schmidt, H. (2006). Systems biology toolbox for MATLAB. www.sbtoolbox.org/.

Schmidt, H., \& Jacobsen, E. W. (2004). Identifying feedback mechanisms behind complex cell behaviors. IEEE Control Systems Magazine, 24, 91102.

Schmidt, H., \& Jirstrand, M. (2006). Systems biology toolbox for MATLAB: A computational platform for research in systems biology. Bioinformatics, 22, 514-515.

Schitztler, A., \& Gross, J. (2005). Open systems in physics and biology. Nature Reviews. Neuroscience, 6, 285-295.

Seehafer, K. (1999). Johann Wolfgang von Goethe: Poet, scientist and statesman. Inter Nationes.

Sharan, R., Ulitsky, I., \& Shamir, R. (2007). Network-based prediction of protein function. Molecular Systems Biology 3(88).

Sontag, E. D. (2005). Molecular systems biology and control. European Journal of Control, 11, 1-40.

Spencer, K. M., Nestor, P. G., Niznikiewicz, M. A., Salisbury, D. F., Shenton, M. E., \& McCarley, R. W. (2003). Abnormal neural synchrony in schizophrenia. The Journal of Neuroscience, 23(19), 7407-7411.

Stelling, J., Sauer, U., Doyle, F. J., \& Doyle, J. (2004). Robustness of cellular function. Cell, 118, 675-685.

Sterling, P. (2004). Principles of allostasis: Optimal design, predictive regulation, pathophysiology and rational therapeutics. In J. Schulkin (Ed.), Allostasis, homeostasis, and the costs of adaptation. Cambridge University Press.

Strange, K. (2005). The end of naive reductionism: The rise of systems biology or renaissance of physiology. American Journal of Physiology. Cell Physiology, 288, 968-974.

Stumpf, M., Wiuf, C., \& May, R. (2005). Subnets of scale-free networks are not scale-free: Sampling properties of networks. Proceedings of the National Academy of Sciences, 102(12), 4221-4224.

Stan, G.-B., \& Sepulchre, R. (2007). Analysis of interconnected oscillators by dissipativity theory. IEEE Transactions on Automatic Control, 52, 256-270.

Sutherland, S. (Ed.). (1992). Irrationality. Pinter and Martin.

The Mathworks. (in press). Simbiology 2.2. www.mathworks.com/products/ simbiology.

The Royal Society. (September 2005). Personalised medicine: Hopes and realities. Technical report, The Royal Society.

Tortura, G. J., \& Grabowski, S. R. (2003). Principles of anatomy and physiology: Control systems of the human body. John Wiley and Sons.

Turner, T. E., Schnell, S., \& Burrage, K. (2004). Stochastic approaches for modelling of in vivo reactions. Computational Biology and Chemistry, 28, $165-178$.

Tyson, J. J., Chen, K. C., \& Novak, B. (2003). Sniffers, buzzers, toggles and blinkers: Dynamics of regulatory and signaling pathways in the cell. Current Opinion in Cell Biology, 15, 221-231.

Valencia, A., \& Pazos, F. (2002). Computational methods for the prediction of protein interactions. Current Opinion in Structural Biology, 12, 368-373. 
Varela, F., Lachaux, J. P., Rodriguez, E., \& Matinerie, J. (2001). The brainweb: Phase synchronization and large-scale integration. Nature Reviews. Neuroscience, 229-239.

Varma, A., \& Palsson, B. O. (1994). Metabolic flux balancing. Biotechnology, 12, 994-998.

Vera, J., Balsa-Canto, E., Wellstead, P., Banga, J. R., \& Wolkenhauer, O. (in press). Power-law models of signal transduction pathways. Cellular Signalling.

Vidyasagar, M., Khammash, M., \& Tomlin, C. (Eds.). (January 2008). Joint special issue on systems biology. IEEE Transactions on Automatic Control \& IEEE Transactions on Circuits and Systems.

Voigt, C. A., Wolf, D. M., Arkin, A. P. (2006). The Bacillus subtilis sin operon: An evolvable network motif. Genetics, 169, 1187-1202.

Wagner, A. (Ed.). (2005). Robustness and evolvability in living systems. Princeton University Press.

Wellstead, P. E. (1979). Physical system modelling. Academic Press.

Weston, A. D., \& Hood, L. (2004). Systems biology, proteomics, and the future of healthcare: Towards predictive, preventative and personalised medicine. Journal of Proteome Research, 3, 179-196.

Wiener, N. (1948). Cybernetics, or control and communication in the animal and the machine. John Wiley and Sons.

Winfree, A. T. (1980). The geometry of biological time. Springer-Verlag.

Wolkenhauer, O., \& Hofmeyr, J.-H. S. (in press). An abstract cell model that describes the self-organisation of cell function in living systems. Journal of Theoretical Biology.

Wolkenhauer, O., Mesarović, M., Sreenath, S., Wellstead, P., \& Rolfs, A. (2006). Regulation. Chapter from regulation, control and adaptation to the coordination of cell function. Vienna series of theoretical biology. Boston: MIT Press.

Wuchty, S., \& Stadler, P. (2003). Centers of complex networks. Journal of Theoretical Biology, 223, 45-53.

Yi, T.-M., Huang, Y., Simon, M. I., \& Doyle, J. (2000). Robust perfect adaptation in bacterial chemotaxis through integral feedback control. Proceedings of the National Academy of Sciences of the United States of America, 97, 4649-4653.

Zhang, L. V., King, O. D., Wong, S. L., Goldberg, D. S., Tong, A. H. Y., Lesage, G., et al. (2005). Motifs, themes and thematic maps of an integrated Saccharomyces cerevisiae interaction network. Journal of Biology, 4, 6.

Peter Wellstead received his B.Sc. degree in electrical engineering from Hatfield College, Hertfordshire, England, and the degree of Ph.D. from Warwick University, Warwickshire, England. He was previously professor of control engineering at the Control Systems Centre, UMIST, Manchester, before taking up his current position in 2004 as Science Foundation Ireland research professor of systems biology at the Hamilton Institute, NUI Maynooth, Co. Kildare in the Republic of Ireland. His current research is in systems biology, which he characterises as the use of mathematical and technological methods developed for the physical sciences to the understanding of mechanisms of life. His research focus is upon a systems approach to disease-specifically Parkinson's Disease. Wellstead is the author of 'Introduction to Physical Systems Modelling' (Academic Press, 1979) and co-author (with M.B. Zarrop) of 'SelfTuning Systems: Control and Signal Processing' (John Wiley, 1991), plus over 150 journal conference articles and numerous contributions to edited volumes.
He is named inventor on several patents and is the originator and chief designer of one of the most comprehensive and widely used ranges of commercially available control engineering teaching equipment.

Eric Bullinger received his diploma degree and the Ph.D. degree in electrical engineering from the ETH Zurich in 1995 and 2001. Since 2007, he is senior lecturer in the Industrial Control Centre, Department of Electronic and Electrical Engineering of the University of Strathclyde in Glasgow, UK. From 2001 to 2005, he was assistant professor at the University of Stuttgart, Germany, in the Institute for Engineering Cybernetics. From 2005 to 2007, he was a research fellow in the Systems Biology group of the Hamilton Institute of the National University of Ireland Maynooth, where he is currently a visiting senior lecturer. His research interests are system identification, observer design and robustness analysis. His is also active in systems biology, from data analysis over modelling to model analysis. He has 13 papers published in peer-reviewed control and biological journals.

Dimitrios Kalamatianos obtained his Diploma of Electrical and Computer Engineering from the University of Patras, Greece, in 2001. He received his $\mathrm{Ph} . \mathrm{D}$. degree in Electrical Engineering and Electronics from the University of Manchester Institute of Science and Technology (UMIST), UK, in 2005. His research involved the development of a novel near-infrared spectrometer for clinical applications and it was supported by the Engineering and Physical Sciences Research Council (EPSRC). He is currently a research fellow at the Hamilton Institute at the National University of Ireland Maynooth, Ireland. His research interests include the application of advanced signal and image processing techniques to biomedical images towards the extraction of quantitative and dynamic information, and the use of control theory for the analysis of in silico models for the understanding of cell death and neural plasticity.

Oliver Mason studied mathematics at Trinity College Dublin (TCD), and was awarded a first class honours degree and a gold medal in his final examinations in 1995. He won several prizes as an undergraduate, including the Townsend Exhibition, Rowe Prize, Minchin Prize, The Lloyd Exhibition and was elected a foundation scholar of the college in 1993. He obtained an M.Sc. degree in mathematics by research from TCD in 1998 and in 2004 a Ph.D. on the stability of switched linear systems from the National University of Ireland Maynooth (NUIM). Currently, his major research interests include the biological network analysis and the applications of graph theory to biology, and the use of novel Lyapunov-based methods to investigate the stability of positive dynamical systems. He has co-authored 16 peer-reviewed journal papers and a similar number of conference papers.

Mark Verwoerd He received his M.Sc. and Ph.D. degree in electrical engineering from The University of Twente, Enschede, The Netherlands, in 2000 and 2005, respectively. Since 2005 he has been a member of staff of The Hamilton Institute, National University of Ireland in Maynooth, Ireland. His research interests are in the general area of systems and control theory, with applications in Biology and Mechatronics. The focus of his current work is on models of biochemical oscillators and synchronization phenomena in biological systems. 